\title{
Co-barcoded sequence reads from long DNA fragments: a cost-effective solution for "perfect genome" sequencing
}

\author{
Brock A. Peters ${ }^{1,2}$, Jia Liu' and Radoje Drmanac ${ }^{1,2}$ * \\ 'Department of Research, Complete Genomics Inc., Mountain View, CA, USA \\ ${ }^{2} B G I$, Shenzhen, China
}

\section{Edited by:}

Yih-Horng Shiao, United States Patent Trademark Office, USA

\section{Reviewed by:}

Michael R. Crowley, University of Alabama at Birmingham, USA

Yih-Horng Shiao, United States Patent Trademark Office, USA

Naoki Osada, National Institute of

Genetics, Japan

\section{${ }^{*}$ Correspondence:}

Brock A. Peters and Radoje Drmanac, Department of Research, Complete Genomics Inc., 2071 Stierlin Court, Mountain View, CA 94043, USA e-mail: bpeters@completegenomics. com; rade@completegenomics.com
Next generation sequencing (NGS) technologies, primarily based on massively parallel sequencing, have touched and radically changed almost all aspects of research worldwide. These technologies have allowed for the rapid analysis, to date, of the genomes of more than 2,000 different species. In humans, NGS has arguably had the largest impact. Over 100,000 genomes of individual humans (based on various estimates) have been sequenced allowing for deep insights into what makes individuals and families unique and what causes disease in each of us. Despite all of this progress, the current state of the art in sequence technology is far from generating a "perfect genome" sequence and much remains to be understood in the biology of human and other organisms' genomes. In the article that follows, we outline why the "perfect genome" in humans is important, what is lacking from current human whole genome sequences, and a potential strategy for achieving the "perfect genome" in a cost effective manner.

Keywords:WGS, whole genome sequencing, haplotyping, NGS, MPS, "perfect genome," LFR, de novo assembly

\section{INTRODUCTION}

Next generation sequencing (NGS) technologies, primarily based on massively parallel sequencing (MPS; Drmanac and Crkvenjakov, 1990; Shendure et al., 2005; Bentley et al., 2008; Drmanac etal., 2010), have touched and radically changed almost all aspects of research worldwide. These technologies have allowed for the rapid analysis, to date, of the genomes of more than 2,000 different species (http://www.genomesonline.org/statistics). In humans, NGS has arguably had the largest impact. Over 100,000 genomes of individual humans (based on various estimates) have been sequenced allowing for deep insights into what makes individuals and families unique and what causes disease in each of us (Rios et al., 2010; Roach et al., 2010; Kong et al., 2012; MacArthur et al., 2012; Michaelson et al., 2012; O’Roak et al., 2012; Sanders et al., 2012; Tennessen et al., 2012; Veltman and Brunner, 2012; Epi et al., 2013; Schaaf et al., 2013; Yang et al., 2013; Al Turki et al., 2014; Fromer et al., 2014; Gilissen et al., 2014; Lawrence et al., 2014; Purcell et al., 2014).

Massively parallel sequencing technologies continue to advance in efficiency with recent improvements allowing "single pixel per spot" imaging on patterned DNA arrays to generate many terabases of read data per sequencing run (Peters et al., 2012) and the recent introduction of Illumina's HiSeq X, also using patterned arrays, producing over $500 \mathrm{~Gb}$ per day. By our estimates, a further several-fold increase in MPS efficiency will be achieved in the next few years, including a reduction in the cost to approximately $\$ 1 / \mathrm{Gb}$ of raw reads. Despite all of this progress, the current state of the art in sequence technology is far from perfect and much remains to be understood in the biology of human and other organisms' genomes. Further, before large-scale adoption of whole genome sequencing (WGS) in humans as a standard of clinical practice can be realized, the quality along multiple different metrics must improve several fold to several orders of magnitude. These improvements can potentially lead to practically complete and errorless reading of individual human genomes. In the article that follows, we outline why this "perfect" WGS ("perfect genome") in humans is important, what is lacking from current human whole genome sequences, and a potential strategy for achieving the "perfect genome" in a cost-effective manner.

\section{THE BENEFITS OF BEING PERFECT}

Within the approximately three billion DNA letters inherited from each parent and the sixteen thousand maternally inherited mitochondrial letters (about 6 billion total) exists a program for the development and adaptive functioning of all of our tissues. As such, it is reasonable to believe that perfect analysis of the human genome will become the ultimate genetic test (Green et al., 2011; Drmanac, 2012) for the discovery of all medically and otherwise relevant variants. Moreover, DNA sequencing of the inherited genome can be administered once and utilized for the entire life of an individual. At the level of a single individual cell there can be many changes in the genome, but averaged over millions of cells in multiple tissues, and excluding special circumstances such as mosaicism or other events that can cause a few dominant clonal genomes, there is little change in a person's genome over a life time. There are important exceptions to this, such as the detection of somatic mutations causing diseases such as cancer where periodic genome sequencing would need to be done on tissue biopsies or blood samples. Coupling perfect WGS with a complete database of genomic variants and gene networks and their corresponding phenotypes will allow for a powerful human health prediction and disease prevention tool. The importance of perfect WGS is analogous to the obvious benefits of errorless reading of computerstored human-generated information. Error-prone retrieval of 
data files would have greatly limited the value of information technologies and significantly impacted their wide use in every day life. Because nuclear and mitochondrial genomes comprise the complete genetic information, perfect sequencing of millions of human genomes per year will form a new irreplaceable infrastructure (similar to the Internet infrastructure) for growing many industries (health, pharmaceuticals, food, sports, and education) with the ultimate goals of improving and extending human life.

\section{WHAT IS PERFECT?}

Perfect WGS or the "perfect genome" is free of errors, meaning there is no need to validate medically relevant variants using some orthogonal sequencing method. DNA bases are not skipped nor are additional bases added in a perfectly read genome and we can be confident that everything that could affect the health of the individual, including all de novo mutations, has been found. In perfect WGS, all deletions, duplications, and disease-causing repeat expansions are detected and the length of each of the 92 telomeres and 24 centromeres is accurately measured. Perfect WGS requires the separate assembly of each parental chromosome (i.e., full haplotype phasing) allowing for the context of all genomic variations to be understood. Contextual interpretation of the influence of genomic variants upon each other is critical for complete understanding of disease predisposition. Even a low false positive or negative error rate would impede any extensive contextual interpretation of genomic variants. Furthermore, a perfectly read genome allows for the full understanding of the complex interactions of genetic and environmental factors. Finally, perfect WGS must be cost effective, ideally less than 1,000 US dollars, otherwise it will be too expensive to be broadly utilized. In practice, it may be difficult to ever achieve a truly "perfect genome," but a genome with a few errors and some unresolved repeat sequences is very obtainable and for the purposes of improving human health, it is exactly what is required. This practically "perfect genome" is what we refer to in the remainder of this article.

\section{FAR FROM PERFECT WHAT'S MISSING?}

Over half of the human genome is composed of repetitive sequence elements (de Koning et al., 2011). The most popular and costeffective sequencing technologies are currently limited to read lengths less than a few 100 base pairs and mate-pair distances typically less than several kilobases; those technologies with longer read lengths are currently prohibitively expensive for whole human genome sequencing or still have error rates too high for routine use. These read architectures allow for sequencing through many repeats, but some repetitive regions are still too long and thus unmappable. Approximately $5-10 \%$ of the human genome falls into these regions that are too long to be analyzed by current technologies. Additionally, some copy number variants (CNVs), especially extra copies of genomic regions $<100 \mathrm{~kb}$ in length and genes with multiple functional or nonfunctional (e.g., pseudogenes) copies can be difficult to analyze accurately. To further complicate matters, current sequencing technologies struggle with regions of extreme AT or GC base content, some of which are important coding and regulatory sequences. Finally, many rare variants, such as de novo mutations, long insertions or deletions, and variants having low read coverage due to various biases are not detected due to the preference to call reference bases or known variants in a reference-based genome assembly.

\section{HUMANS ARE DIPLOID}

Most individuals have more than 2 million locations in their genome where the 22 autosomal chromosomes inherited from each parent differ (Levy et al., 2007; Bentley et al., 2008; Wang et al., 2008; Wheeler et al., 2008; Ahn et al., 2009; Drmanac et al., 2010; Peters et al., 2012). These variants can have profound effects on the biology of the individual depending on if they are found on the same chromosome or opposite homologous chromosomes. Read lengths of a few hundred base pairs or less are insufficient to determine which combination of variants exists on a single parental chromosome. As a result most whole genome assemblies are haploid consensus in nature with both variants listed at each genomic position where a difference is found, but little to no information about the parental chromosome from which they came. For example, in the case where two detrimental variants are found in a single gene it is imperative to know if at least one of the parental genes is functional for genetic diagnosis. For imprinted genes a single detrimental variant can be disease-causing depending on the parental chromosome on which it resides (Schaaf et al., 2013).

\section{FINDING A SIGNAL IN THE NOISE}

Next generation sequencing has begun to become more prevalent in the clinical setting, but false positive error rates are still too high [one false positive error in every 1 million bases (Roach et al., 2010)] for WGS to become routine clinical practice. False positive errors cloud the already difficult analysis of WGS data, creating potentially misleading detrimental variants that require additional analysis work or sequence validation to confirm. Many of these errors come from mismapping sequence reads between genomic regions evolved by duplication or repeat multiplication. Of equal importance are those variants that are missed in regions that are otherwise called or variants removed by filters during the genome assembly process to reduce false positive errors. All clinical tests suffer from these false positive and negative errors, however, WGS has much room to improve on these dimensions.

\section{LESS IS MORE}

Thousands of cells are not always available in a clinical setting, but that is the amount of DNA required by most WGS technologies. Ideally WGS could be performed on 10 cells or less. This would allow for the application of WGS to in vitro fertilized embryo biopsies (Peters et al., under review Genome Research), circulating tumor cells, circulating or other fetal cells (Bolnick et al., 2014), and other biological samples with a small number of cells available. Starting with a small amount of DNA (66 pg in 10 human cells) requires DNA amplification that introduces false positive errors and other artifacts and biases making perfect WGS from a few cells especially challenging.

\section{TOWARD PERFECTION}

Our "perfect genome" solution employs advanced massively parallel DNA sequencing of "co-barcoded" reads from long genomic DNA molecules, and efficient de novo assembly empowered by 


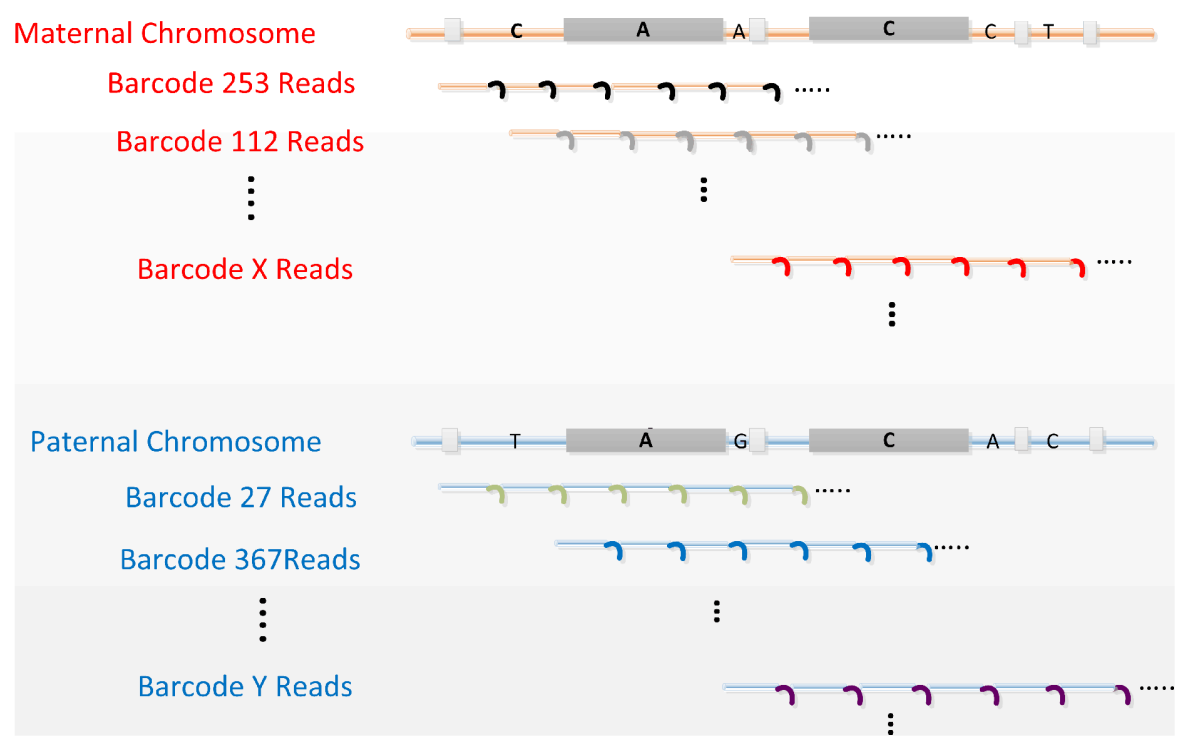

FIGURE 1 | The concept of read co-barcoding for advanced whole genome sequencing (WGS). All four critical requirements are depicted. (1) A genomic library is prepared from long DNA (e.g., 30-300 kb) representing 10 or more cells. Multiple staggered long DNA fragments for each genomic region are generated as a result of random fragmenting during cell lysis (three fragments depicted under each parental chromosome). In the co-barcoded read libraries these redundant long fragments allow variant phasing, a more accurate assembly of the genome, and ultimately de novo assembly. In this example a pair of long proximate repeat elements, longer than the read and mate-pair length, is shown by the large gray boxes. $A$ and $C$ denote single base differences between copies of these repeat elements. Long, overlapping, staggered genomic fragments allow for the proper placement of these repeats in the final assembly by exclusive linking of repeat members to surrounding unique sequences provided by the long DNA fragments that start or end between repeats. (2) Sequence reads generated from each long fragment (i.e., subfragments used to produce these reads) are tagged (small colored curved lines) with the same barcode (co-barcoded). There are many (usually 10s-100s) of reads per long DNA fragment, most if not all having the same barcode. Reads belonging to related (i.e., overlapped) long fragments mostly have different barcodes. Consequently maternal (red) and paternal (blue) fragments for a genomic region have different barcodes as indicated by the distinct barcode numbers $(253,112$, and $X$ for mom, 27, 367, and $Y$ for dad). After MPS, barcodes are used to aggregate reads from the original long fragment. Such read aggregation, even without sequence assembly per long fragment, provides information for variant phasing and repeat resolving when reads from overlapping long fragments, representing the same chromosome, are used together in the assembly process. (3) Sequence reads must cover $>30 \%$ and preferably the majority of bases in each long fragment. Consecutive continuous reads (depicted here) or overlapping mate-pair reads (two shorter reads from the ends of the same subfragment) can provide the needed coverage. Sequencing the majority of bases of each fragment with co-barcoded reads links alleles in haplotypes as, on average, 10 or more heterozygous sites occur per long DNA fragment. (4) The read or mate-pair length is longer than the frequent dispersed repeats (e.g., Alu, depicted by the small gray boxes) and are correctly assembled primarily using read level data. these barcoded reads. The critical requirements of this fundamental and comprehensive solution for sequencing complex diploid genomes are depicted in Figure 1.

First, a sample providing sufficient (e.g., >10X) genome coverage in long (e.g., 30-300 kb) DNA fragments is needed. This redundancy allows the differentiation of true sequences from errors and artifacts and the separation of long clustered repeats (e.g., full length LINEs or segmental duplications) as discussed below. Implicit in this requirement is DNA isolated from at least several cells from one individual and the preservation of long DNA molecules. This eliminates formalin-fixed paraffinembedded (FFPE) samples, in which DNA is typically degraded, and single cells as sources for our "perfect genome."

Second, nearly all reads from a long fragment must share the same unique barcode ("co-barcoding") and that barcode must be different from the barcodes used to tag reads from the majority of (i.e., $>80 \%$ ) related long DNA fragments (e.g., overlapped DNA fragments covering a genomic region or copies of long repeats). However, reads from many unrelated long fragments can have the same barcode.
Third, sufficient base coverage from each long DNA fragment (at least $\sim 30 \%$ and preferably $>60 \%$ of bases with read coverage per fragment between $0.5 \mathrm{X}$ and $2 \mathrm{X}$ ) is needed to link most of the sequence belonging to a long fragment and achieve almost all the benefits of very long reads (i.e., $>50 \mathrm{~kb}$ ). In the case of ideal unbiased WGS, a total read coverage of $40 \mathrm{X}$ would be sufficient for 10 diploid cells with co-barcoding, although in practice over $100 \mathrm{X}$ would be preferred.

Fourth, a read structure, as discussed below, that allows for the resolution of frequent short repeats (e.g., Alu and similar repeats) and the ability to measure the length of homopolymers and tandem repeats is important.

It is critical to satisfy all four listed requirements in this optimally designed arrangement in such a manner that DNA tagging does not add significantly to the total cost.

\section{DEMONSTRATION OF CO-BARCODED READS}

The principles behind co-barcoded reads (Figure 1) have previously been described (Drmanac, 2006) and an experimental implementation of these principles called long fragment read 
technology (LFR; Peters et al., 2012) has been developed. Briefly, long related DNA fragments (e.g., overlapped fragments from a genomic region) are separated stochastically by pipetting a dilute high molecular weight DNA solution into physically distinct wells, followed by multiple displacement amplification (MDA; Dean etal., 2002) and fragmentation to generate shorter molecules (subfragments) suitable for short read mate-pair sequencing. Barcoded adapter molecules, unique to each well, are then ligated to the subfragments. Finally, the barcoded DNA from all wells is combined and sequenced, including reading the 10-base barcodes. All reactions preceding pooling are performed in the same plate without any DNA purification between steps enabling use of a large number of small wells. The initial dilution and separation of long DNA molecules is done such that approximately $10 \%$ of a haploid genome $(\sim 300 \mathrm{Mb})$ is found in each well. Statistically this results in a $5 \%$ chance that for a given region of the genome overlapping molecules from each parent will be present in the same well. When performed in a 384-well plate there is a redundancy of $\sim 20$ long fragments from each parent $(\sim 40$ in total), for any position in the genome, distributed across 36 wells on average. This results in each genomic location having DNA exclusively from one parent in 17 (2 of 36 wells have mixed parents) wells, on average, with an overall range of 6-20 wells.

\section{A LARGE NUMBER OF BARCODES IS CRITICAL FOR PERFECT WGS}

Co-barcoding, as previously demonstrated (Peters et al., 2012), allows for haplotyping and substantial error correction, making it a key part of working toward the "perfect genome." However, 384 wells do not allow for efficient de novo assembly and as will be discussed later, de novo assembly is absolutely critical for a "perfect genome." The problem is that with $10 \%$ of the genome labeled by each barcode, and the repetitive nature of the human genome, there are too many overlapping short reads from distinct genomic regions in each well. The obvious way to improve this is to increase the number of physically distinct compartments and thus the number of barcodes, which reduces the percent of a haploid genome for each compartment.

How many barcodes are needed? The more the better, but to be able to measure common long repeats such as telomeres from 20 cells ( $\sim 2,000$ telomeres) we would need at least 4,000 compartments (providing $>75 \%$ isolated telomeres). This number of compartments is also needed to accurately count mitochondrial genomes and assess heteroplasmy as there are often more than 100 mitochondria per cell. Therefore, $5,000-10,000$ is a good starting point. Using 10,000 individual compartments with 10,000 unique barcodes and 20 cells would result in only one hundred and twenty $100 \mathrm{~kb}$ molecules ( $12 \mathrm{Mb}$ of DNA) per barcode. This small number of bases per each barcode dramatically reduces the chance of co-barcoding related long fragments. Additionally, 10,000 barcodes reduces the number of highly similar Alu repeats and LINEs with the same barcode to $<1,000$ for each repeat type. However, while 10,000 barcodes should be sufficient this suggests a larger number would be beneficial for analyzing these highly redundant DNA elements.

With more barcodes it may be more optimal to use more cells, ideally 50 if available, to increase the number of staggered long
DNA fragments covering each genomic region. This would provide more power to separate long proximate repeats as discussed in Figure 1. More cells would also enable more independent measurements for error reduction (discussed below), assuming more reads are generated. For $1 \mathrm{X}$ read coverage reading $\sim 60 \%$ of bases of each long DNA molecule from 50 cells, 100X total read coverage is needed without any coverage bias. On average, this would result in 30 independent reads per parental base and cost about $\$ 300$ to generate with the projected reduction of sequencing costs to $\$ 1 / \mathrm{Gb}$.

\section{ELIMINATING LONG DNA AMPLIFICATION}

Using 10,000 barcodes and 50 or more cells allows for the possibility to skip amplification of long DNA [e.g., MDA (Dean et al., 2002), MALBAC (Zong et al., 2012), or long range PCR]. Current long DNA amplification strategies introduce coverage bias and, if PCR is used (Kuleshov et al., 2014) limit fragment length to less than $10 \mathrm{~kb}$. Ultimately, this amplification bias requires additional read coverage, $200 \mathrm{X}$ or more, to assemble a complete genome if there are no regions with severe under-amplification. Libraries barcoded prior to any amplification would result in nonoverlapping adjacent co-barcoded 500-1,500 bp subfragments generated from each long DNA molecule. Using current MPS equipment would result in only the ends of each subfragment being read, and thus a lower fraction of bases per long fragment would be interrogated $(\sim 30 \%)$. This would significantly complicate analysis and most likely would require improved read architecture as discussed later. Otherwise, minimal long DNA amplification of 10-100X may provide a good balance between the fraction of bases read per fragment and coverage bias.

\section{HOW LONG SHOULD LONG FRAGMENTS BE?}

Long DNA fragments, over $1 \mathrm{Mb}$ in some cases, are needed to phase across long genomic regions with no or a few heterozygous loci commonly found in the genomes of non-African populations (Peters et al., 2012) or to assess centromeric regions. However, for resolving intermediate repeats, such as LINEs, that are longer than mate-pair lengths random pools of one hundred approximately $100 \mathrm{~kb}$ fragments are more valuable than single $10 \mathrm{Mb}$ fragments (Figure 1). An optimal solution is a broad distribution of fragment lengths from $30 \mathrm{~kb}$ to $3 \mathrm{Mb}$ prepared from at least 50 cells, potentially using multiple different methods (e.g., enzymatic and mechanical) to improve the randomness of fragment ends. This would provide both frequent starts of fragments and a sufficient number of very long fragments (i.e., $3 \mathrm{Mb}$ ). This could be achieved by splitting cells into separate reactions prior to lysis and then performing different amounts of DNA fragmenting on each reaction. To preserve very long DNA, a reversible chromatin crosslinking process could potentially be used (de Vree et al., 2014).

\section{CAN WE USE 10,000 BARCODES COST-EFFECTIVELY?}

Five thousand to 10,000 individual DNA aliquots is achievable with current technologies using our multi-step no-purification biochemical protocol (Peters et al., 2012) and there are companies (e.g., WaferGen, Fluidigm, etc.) selling devices that can dispense pico to nanoliters of liquid into microwells that can accommodate 
total volumes up to several hundred nanoliters. Alternatively, over 100,000 compartments can be generated easily in less than a minute using currently available emulsion based approaches (e.g., Raindrop, Bio Rad, etc.) and combinatorial barcoding in nanodrops (Drmanac et al., 2011). As these technologies improve the cost of using them will drop to become less than the cost of a 384well plate. Importantly, if nanoliter or picoliter volume aliquots are used, the cost of reagents will be less than our current cobarcoding process $(\sim \$ 50$, several microliters per aliquot; Peters et al., 2012).

\section{WHAT READ STRUCTURE IS BEST?}

Current MPS read lengths of about 50 bases or more are suitable for our proposed "perfect genome" strategy. However as discussed above, long DNA amplification will most likely be necessary if this read architecture is used. If an approach without long DNA amplification proves to be beneficial, a feasible near-term solution could be improved paired-end MPS with $\sim 300$ base reads from each end of 500-1,500 bp subfragments (including the barcode sequence). Cost-effective and scalable single molecule sequencing of more than 500 bases would be even better if and when it becomes available. These longer paired-end or single reads could improve the resolution of repeats in close proximity to each other that are longer than current reads but too short to be resolved by the mate-pair read structure due to variation in the subfragment size (generally varying from 500 to $1,500 \mathrm{bp}$ ). Furthermore, longer reads reduce the negative effects of non-randomness from DNA fragmenting. 500 base reads would also provide a more precise measure of long triple and other tandem repeats. In our proposed co-barcoding based strategy, longer continuous reads (e.g., $5 \mathrm{~kb}$ ) are less beneficial, especially if they are error prone and/or more expensive.

\section{ERROR FREE REFERENCE FREE PHASED GENOME ASSEMBLY}

Our low cost "perfect genome" solution requires 10-100 cells and thus has to have a low read coverage per each long fragment (usually less than $2 \mathrm{X}$ ), otherwise it would be very expensive to generate $600 \mathrm{X}$ read coverage $(10$ cells $\times 2$ parental sets of chromosomes $\times 30 \mathrm{X}$ standard read coverage). Therefore, the trivial approach of collecting all reads with the same barcode to assemble long DNA fragments is not applicable. Instead, integrating low read coverage from 10 or more original long overlapped fragments with different barcodes per each genomic region is required. We previously described an approach for variant calling disregarding barcodes and then using barcodes for phasing heterozygous variants by calculating the number of shared compartments for alleles in the neighboring heterozygous loci (Peters etal., 2012). Using this method we also demonstrated that many false positive errors could be removed as it is unlikely for errors to repeatedly occur (i.e., have supporting reads with multiple different barcodes) exclusively on one parental chromosome, and if errors do occur on only one parental chromosome they are typically from a single barcoded fragment. In both cases, these errors can be identified and removed (Peters et al., 2012).

The above approaches are very effective but use a referencebiased process in the initial variant calling. Using a large number of barcodes allows implementation of a computationally and cost efficient (less than $\$ 200$ by our estimates) reference-free or reference-unbiased assembly. Cycle sequencing used in MPS generates reads without indel errors. This allows for the nongapped read-to-read alignments necessary for efficient de novo assembly especially by implementing k-mer-based read-to-read indexing using computers with large RAM (e.g., 4TB) and 80 or more cores. Such non-gapped alignments cannot be used for mapping reads to the reference to call indels. Read-to-read nongaped aligment has previously been implemented in a local de novo assembly (Carnevali et al., 2012). Co-barcoding with 10,000 or more barcodes provides substantial localization of reads for efficient and cost-effective assembly of long de novo contigs. For example, calling high quality heterozygous variants first (Drmanac et al., 2011) allows the segregation of co-barcoded reads to each parental chromosome for each genomic region having heterozygous positions. For most of the genome, this localization and segregation allows de novo assembly of large genomic regions separately for each parental chromosome. Such advanced phased de novo assembly would not only provide variant calling unbiased by the reference sequence, it would almost completely prevent calling sequencing errors or mutations introduced by DNA amplification as heterozygous variants. In this approach, the majority of positions in a genome are called with higher accuracy as two hemizygous calls supported by a sufficient number of exclusive parental barcodes. Phased de novo assembly using co-barcoded reads from long staggered DNA fragments, especially from $\sim 50$ cells, allows the resolution of variants in complex gene families including hemizygous deletions smaller than an exon. For detection and enumeration of CNVs, counting the number of different barcodes per genomic region separately for each parent allows for a more precise measurement than read counts which are much more prone to various biases. Combined with de novo assembly, that defines CNVs by sequence junctions, this advanced LFR process can provide a more complete and accurate genome sequence. Developing advanced co-barcoding and efficient reference unbiased de novo assembly of individual human genomes is of critical importance for the broad and efficient implementation of genomic medicine.

\section{PUTTING IT ALL TOGETHER}

In this manuscript we have outlined a proposal for achieving the "perfect genome" sequence using current and yet to be developed technologies. In Figure 2 we summarize our preferred end-toend strategy to reach this goal. Almost all parts of the process are currently available but substantial optimization is needed, especially in making representative co-barcoded sequencing libraries in thousands of nano-wells without long DNA amplification and in developing sophisticated phased de novo assembly software. Indeed, a recently published paper by Adey et al. (2014) has shown that co-barcoding with $\sim 10,000$ barcodes allows for substantial de novo assembly without long DNA amplification. However, due to very low read coverage per long fragment, violating one of the four critical requirements for perfect WGS by co-barcoded reads, a large number of cells $(\sim 500)$ are required. This results in about $5-10 \%$ of a haploid genome per barcode, approximately $10 \mathrm{X}$ more than we are proposing. Further, their method is unable to achieve 


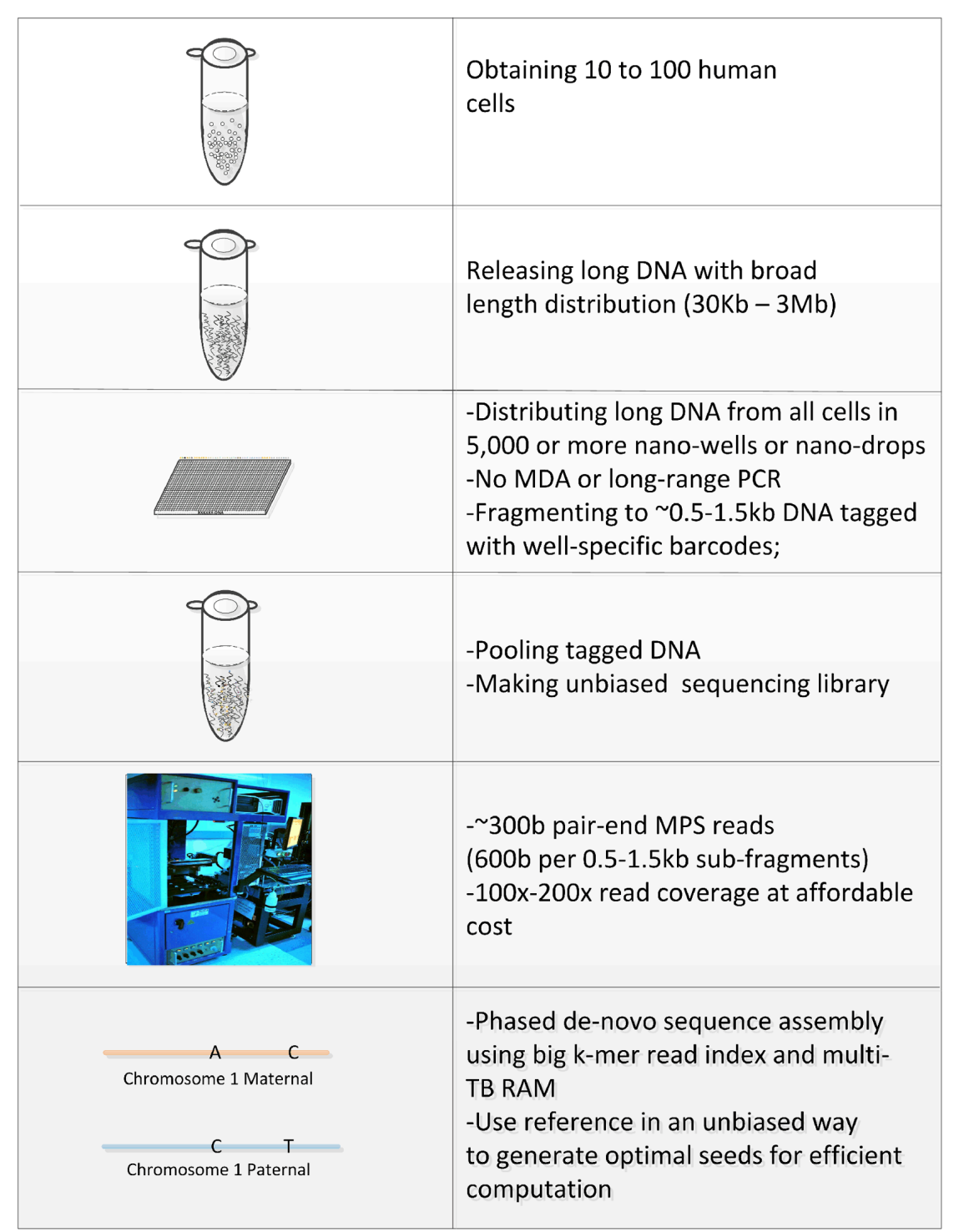

FIGURE 2 | Integrated "perfect genome" solution. Our preferred end-to-end proposal for achieving the perfect genome is summarized in this table. The "perfect genome" starts with an input of 10-100 unfixed cells. Cells are lysed and long DNA fragments with a broad length distribution are released and divided into 5,000 or more compartments. Subfragments 500-1,500 bp in length are generated, without long DNA amplification, and labeled with a barcode specific to each compartment (co-barcoded). All compartments are pooled into a single tube and advanced pair-end MPS using 300 base reads is performed, including reading 10 bases or longer barcode sequences. Reference unbiased phased de novo assembly of each parental chromosome is performed using k-mer read indexes on large terabyte RAM servers. Obtaining the "perfect genome" from a smaller number of cells (e.g., 10-20) would require maximal optimization of all steps. substantial phasing and as such the result is still far from a "perfect genome."

The most important part of our proposal that is not currently available is a high quality, affordable 300 base MPS paired-end read length (a total of 600 bases read per subfragment). However, given the dramatic improvement in NGS read lengths over the past few years, we believe 300 base reads will be available soon. As discussed, a 10-100 fold low-bias amplification of long DNA coupled with 100 base reads is an alternative solution if 300 base reads are not feasible or cost-effective. It should be noted that while we focused on human genome sequencing for this article, the strategy outlined here is a viable solution for analyzing the genomes of any species.

\section{CONCLUSION}

Improvements to MPS-based NGS continue to advance our ability to efficiently analyze a large number of individual human genomes with high sensitivity and specificity, including separate sequence assembly of parental chromosomes (haplotyping) with recently reported false positive SNV errors of less than 10 per human genome sequenced from a 10-cell sample [Peters et al., under review Genome Research]. Co-barcoded reads with at least 
5,000 barcodes and advanced phased sequence assembly unbiased by the human reference genome, as proposed here, will further improve accuracy and completeness and allow for the achievement of the "perfect genome." This type of low cost, high throughput solution promises to enable the efficient and accurate sequence analysis of the entire genome of millions of people as well as from difficult to analyze samples such as micro-biopsies of in vitro fertilized embryos and fetal and tumor cells collected from the blood. This ultimate genetic test, when applied broadly, will provide a foundation for genomics-based precision medicine and disease prevention through "genomic healthcare"; enabling a longer and healthier life.

\section{ACKNOWLEDGMENTS}

We would like to acknowledge the ongoing contributions and support of all Complete Genomics, Inc. employees, in particular the many highly skilled individuals that work in the libraries, reagents, and sequencing groups that make it possible to generate high quality whole genome data.

\section{REFERENCES}

Adey, A., Kitzman, J. O., Burton, J. N., Daza, R., Kumar, A., Christiansen, L., et al. (2014). In vitro, long-range sequence information for de novo genome assembly via transposase contiguity. Genome Res. 24, 2041-2049. doi: 10.1101/gr.178319.114

Ahn, S. M., Kim, T. H., Lee, S., Kim, D., Ghang, H., Kim, D. S., et al. (2009). The first Korean genome sequence and analysis: full genome sequencing for a socio-ethnic group. Genome Res. 19, 1622-1629. doi: 10.1101/gr.092197.109

Al Turki, S., Manickaraj, A. K., Mercer, C. L., Gerety, S. S., Hitz, M. P., Lindsay, S., etal. (2014). Rare variants in NR2F2 cause congenital heart defects in humans. Am. J. Hum. Genet. 94, 574-585. doi: 10.1016/j.ajhg.2014. 03.007

Bentley, D. R., Balasubramanian, S., Swerdlow, H. P., Smith, G. P., Milton, J., Brown, C. G., etal. (2008). Accurate whole human genome sequencing using reversible terminator chemistry. Nature 456, 53-59. doi: 10.1038/nature 07517

Bolnick, J. M., Kilburn, B. A., Bajpayee, S., Reddy, N., Jeelani, R., Crone, B., et al. (2014). Trophoblast retrieval and isolation from the cervix (TRIC) for noninvasive prenatal screening at 5 to 20 weeks of gestation. Fertil. Steril. 102, 135-142.e6. doi: 10.1016/j.fertnstert.2014.04.008

Carnevali, P., Baccash, J., Halpern, A. L., Nazarenko, I., Nilsen, G. B., Pant, K. P., et al. (2012). Computational techniques for human genome resequencing using mated gapped reads. J. Comput. Biol. 19, 279-292. doi: 10.1089/cmb. 2011.0201

Dean, F. B., Hosono, S., Fang, L., Wu, X., Faruqi, A. F., Bray-Ward, P., et al. (2002). Comprehensive human genome amplification using multiple displacement amplification. Proc. Natl. Acad. Sci. U.S.A. 99, 5261-5266. doi: 10.1073/pnas.082089499

de Koning, A. P., Gu, W., Castoe, T. A., Batzer, M. A., and Pollock, D. D. (2011). Repetitive elements may comprise over two-thirds of the human genome. PLoS Genet. 7:e1002384. doi: 10.1371/journal.pgen.1002384

de Vree, P. J., De Wit, E., Yilmaz, M., Van De Heijning, M., Klous, P., Verstegen, M. J., et al. (2014). Targeted sequencing by proximity ligation for comprehensive variant detection and local haplotyping. Nat. Biotechnol. 32, 1019-1025. doi: $10.1038 /$ nbt.2959

Drmanac, R. (2006). Nucleic acid analysis by random mixtures of non-overlapping Fragments. Patent WO 2006/138284 A2.

Drmanac, R. (2012). Medicine. The ultimate genetic test. Science 336, 1110-1112. doi: $10.1126 /$ science. 1221037

Drmanac, R., and Crkvenjakov, R. (1990). Prospects for a miniaturized, simplified, and frugal human genome project. Sci. Yugoslavica 16, 97-107.

Drmanac, R., Peters, B. A., Alexeev, A., and Hong, P. (2011). Methods and Compositions for Long Fragment Read Sequencing. Patent US 8592150 B2.

Drmanac, R., Sparks, A. B., Callow, M. J., Halpern, A. L., Burns, N. L., Kermani, B. G., et al. (2010). Human genome sequencing using unchained base reads on self-assembling DNA nanoarrays. Science 327, 78-81. doi: 10.1126/science. 1181498

Epi, K. C., Epilepsy Phenome/Genome, P., Allen, A. S., Berkovic, S. F., Cossette, P., Delanty, N., et al. (2013). De novo mutations in epileptic encephalopathies. Nature 501, 217-221. doi: 10.1038/nature12439

Fromer, M., Pocklington, A. J., Kavanagh, D. H., Williams, H. J., Dwyer, S., Gormley, P., et al. (2014). De novo mutations in schizophrenia implicate synaptic networks. Nature 506, 179-184. doi: 10.1038/nature12929

Gilissen, C., Hehir-Kwa, J. Y., Thung, D. T., Van De Vorst, M., Van Bon, B. W., Willemsen, M. H., et al. (2014). Genome sequencing identifies major causes of severe intellectual disability. Nature 511, 344-347. doi: 10.1038/nature 13394

Green, E. D., Guyer, M. S., and National Human Genome Research, I. (2011). Charting a course for genomic medicine from base pairs to bedside. Nature 470, 204-213. doi: 10.1038/nature09764

Kong, A., Frigge, M. L., Masson, G., Besenbacher, S., Sulem, P., Magnusson, G., et al. (2012). Rate of de novo mutations and the importance of father's age to disease risk. Nature 488, 471-475. doi: 10.1038/nature11396

Kuleshov, V., Xie, D., Chen, R., Pushkarev, D., Ma, Z., Blauwkamp, T., et al. (2014). Whole-genome haplotyping using long reads and statistical methods. Nat. Biotechnol. 32, 261-266. doi: 10.1038/nbt.2833

Lawrence, M. S., Stojanov, P., Mermel, C. H., Robinson, J. T., Garraway, L. A., Golub, T. R., et al. (2014). Discovery and saturation analysis of cancer genes across 21 tumour types. Nature 505, 495-501. doi: 10.1038/nature12912

Levy, S., Sutton, G., Ng, P. C., Feuk, L., Halpern, A. L., Walenz, B. P., et al. (2007). The diploid genome sequence of an individual human. PLoS Biol. 5:e254. doi: 10.1371/journal.pbio.0050254

MacArthur, D. G., Balasubramanian, S., Frankish, A., Huang, N., Morris, J., Walter, K., etal. (2012). A systematic survey of loss-of-function variants in human protein-coding genes. Science 335, 823-828. doi: 10.1126/science. 1215040

Michaelson, J. J., Shi, Y., Gujral, M., Zheng, H., Malhotra, D., Jin, X., et al. (2012). Whole-genome sequencing in autism identifies hot spots for de novo germline mutation. Cell 151, 1431-1442. doi: 10.1016/j.cell.2012.11.019

O'Roak, B. J., Vives, L., Girirajan, S., Karakoc, E., Krumm, N., Coe, B. P., et al. (2012). Sporadic autism exomes reveal a highly interconnected protein network of de novo mutations. Nature 485, 246-250. doi: 10.1038/nature 10989

Peters, B. A., Kermani, B. G., Sparks, A. B., Alferov, O., Hong, P., Alexeev, A., et al. (2012). Accurate whole-genome sequencing and haplotyping from 10 to 20 human cells. Nature 487, 190-195. doi: 10.1038/nature11236

Purcell, S. M., Moran, J. L., Fromer, M., Ruderfer, D., Solovieff, N., Roussos, P., et al. (2014). A polygenic burden of rare disruptive mutations in schizophrenia. Nature 506, 185-190. doi: 10.1038/nature12975

Rios, J., Stein, E., Shendure, J., Hobbs, H. H., and Cohen, J. C. (2010). Identification by whole-genome resequencing of gene defect responsible for severe hypercholesterolemia. Hum. Mol. Genet. 19, 4313-4318. doi: 10.1093/hmg/ ddq352

Roach, J. C., Glusman, G., Smit, A. F., Huff, C. D., Hubley, R., Shannon, P. T., etal. (2010). Analysis of genetic inheritance in a family quartet by whole-genome sequencing. Science 328, 636-639. doi: 10.1126/science.11 86802

Sanders, S. J., Murtha, M. T., Gupta, A. R., Murdoch, J. D., Raubeson, M. J., Willsey, A. J., et al. (2012). De novo mutations revealed by whole-exome sequencing are strongly associated with autism. Nature 485, 237-241. doi: 10.1038/nature 10945

Schaaf, C. P., Gonzalez-Garay, M. L., Xia, F., Potocki, L., Gripp, K. W., Zhang, B., et al. (2013). Truncating mutations of MAGEL2 cause Prader-Willi phenotypes and autism. Nat. Genet. 45, 1405-1408. doi: 10.1038/ng.2776

Shendure, J., Porreca, G. J., Reppas, N. B., Lin, X., Mccutcheon, J. P., Rosenbaum, A. M., et al. (2005). Accurate multiplex polony sequencing of an evolved bacterial genome. Science 309, 1728-1732. doi: 10.1126/science.1117389

Tennessen, J. A., Bigham, A. W., O'connor, T. D., Fu, W., Kenny, E. E., Gravel, S., et al. (2012). Evolution and functional impact of rare coding variation from deep sequencing of human exomes. Science 337, 64-69. doi: 10.1126/science. 1219240

Veltman, J. A., and Brunner, H. G. (2012). De novo mutations in human genetic disease. Nat. Rev. Genet. 13, 565-575. doi: 10.1038/nrg3241 
Wang, J., Wang, W., Li, R., Li, Y., Tian, G., Goodman, L., et al. (2008). The diploid genome sequence of an Asian individual. Nature 456, 60-65. doi: 10.1038 /nature07484

Wheeler, D. A., Srinivasan, M., Egholm, M., Shen, Y., Chen, L., Mcguire, A., et al. (2008). The complete genome of an individual by massively parallel DNA sequencing. Nature 452, 872-876. doi: 10.1038/nature06884

Yang, Y., Muzny, D. M., Reid, J. G., Bainbridge, M. N., Willis, A., Ward, P. A., et al. (2013). Clinical whole-exome sequencing for the diagnosis of mendelian disorders. N. Engl. J. Med. 369, 1502-1511. doi: 10.1056/NEJMoa1306555

Zong, C., Lu, S., Chapman, A. R., and Xie, X. S. (2012). Genome-wide detection of single-nucleotide and copy-number variations of a single human cell. Science 338, 1622-1626. doi: 10.1126/science.1229164

Conflict of Interest Statement: The authors are employed by Complete Genomics, Inc., a whole human genome sequencing company. Complete Genomics, Inc. is a wholly owned subsidiary of BGI-Shenzhen, a DNA sequencing company. The authors have shares in BGI-Shenzhen. Complete Genomics, Inc. has filed patents on many of the topics discussed in this article.

Received: 08 October 2014; accepted: 18 December 2014; published online: 14 January 2015.

Citation: Peters BA, Liu J and Drmanac R (2015) Co-barcoded sequence reads from long DNA fragments: a cost-effective solution for "Perfect Genome" sequencing. Front. Genet. 5:466. doi: 10.3389/fgene.2014.00466

This article was submitted to Genomic Assay Technology, a section of the journal Frontiers in Genetics.

Copyright (c) 2015 Peters, Liu and Drmanac. This is an open-access article distributed under the terms of the Creative Commons Attribution License (CC BY). The use, distribution or reproduction in other forums is permitted, provided the original author(s) or licensor are credited and that the original publication in this journal is cited, in accordance with accepted academic practice. No use, distribution or reproduction is permitted which does not comply with these terms. 